\title{
Study of the Li-Oxygen Battery Discharging and Charging Process using In-situ TEM
}

\author{
Kun $\mathrm{He}^{1}$, Yifei Yuan ${ }^{1,2}$, Xuanxuan $\mathrm{Bi}^{2}$, Tara Foroozan ${ }^{1}$, Jun $\mathrm{Lu}^{2}$, Reza Shahbazian-Yassar ${ }^{1}$ \\ 1. University of Illinois at Chicago, Department of Industrial and Mechanical Engineering, Chicago, IL, \\ USA \\ 2. Chemical Science and Engineering Division, Argonne National Laboratory, Argonne, IL, USA
}

Nowadays, lithium-oxygen battery is receiving more and more research attention as the promising nextgeneration rechargeable battery system due to its high energy density $(>3500 \mathrm{~W} \cdot \mathrm{h} / \mathrm{kg})[1]$ that is about 10 times higher than that of current lithium-ion batteries. However, the application of lithium-oxygen battery is hindered by several issues such as poor cyclability, limited rate capability and high overpotential during the oxygen reduction reaction (ORR) and the oxygen evolution reaction (OER)[2]. What's worse, the mechanisms of lithium-oxygen battery system are poorly understood due to the highly dynamic nature of this battery system as well as the air-sensitive charge-discharge products that disable most conventional ex situ characterization methods. Consequently, lots of questions are not well answered, such as the formation of complicated discharge products $\left(\mathrm{LiOH}, \mathrm{Li}_{2} \mathrm{O}, \mathrm{Li}_{2} \mathrm{O}_{2} \mathrm{LiO}_{2}\right)$, [3] the detailed nucleation, growth and dissolution processes of lithium oxides during charge and discharge, and the origins for large overpotential.

Here, using in situ transmission electron microscopy (TEM) combined with an electrochemical liquid cell containing liquid electrolyte, the (dis)charge processes of the lithium-oxygen battery are studied in real time. The oxygen-rich electrolyte are flowed inside the sealed liquid cell made of silicon substrate and silicon nitride window for electron transparency. Upon the control of the potential applied to the $\mathrm{LiCoO}_{2}$ cathode and the metal anode, the charging and discharging kinetics are observed on the cathode side. It is found that the discharge (ORR) process on the anode side starts with the formation of $\mathrm{Li}_{2} \mathrm{O}_{2}$ nuclei of tens of nanometers, which are tightly attached to the electrode surface. As the discharge goes on, the $\mathrm{Li}_{2} \mathrm{O}_{2}$ nuclei on electrode keeps growing while more nuclei start to precipitate from the electrolyte not contacting the current collector. Such observation implies that the formation of $\mathrm{Li}_{2} \mathrm{O}_{2}$ starts with the formation of soluble $\mathrm{O}^{2-}$ on the anode side, which is a single-electron electrochemical reaction. As the $\mathrm{O}^{2-}$ diffuse into the electrolyte to make it $\mathrm{O}^{2-}$ rich, the disproportionation reaction (chemical reaction instead of electrochemical) of soluble $\mathrm{O}_{2}^{-}$takes place as the intermediate step to produce $\mathrm{Li}_{2} \mathrm{O}_{2}$ on both the anode surface and the electrolyte. On charge process (OER), the decomposition of $\mathrm{Li}_{2} \mathrm{O}_{2}$ starts at the electrodeelectrolyte interface, which implies that OER is controlled by the electron transport kinetics instead of $\mathrm{Li}^{+}$ diffusion kinetics. The fundamentals discovered in this work reveal the underlying working mechanisms of Li-O $\mathrm{O}_{2}$ electrochemical couples, and they help to account for the practically sluggish ORR and OER reaction kinetics observed in lithium-oxygen batteries as well as to provide guidance for the further improvement of lithium-oxygen battery system.

\section{References:}

[1] Lu, J et al, Chem Rev 114 (2014), p. 5611.

[2] Yang, J et al, Phys Chem Chem Phys 15 (2013), p. 3764.

[3] Lu, J et al, Nature (2016). 
a

b
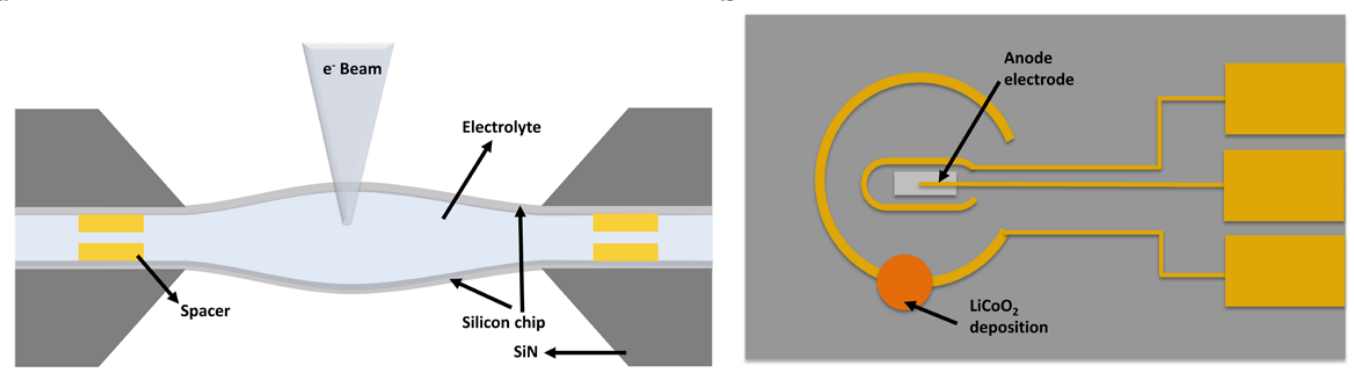

Figure 1. The schematic image of the liquid cell design (a) and the deposition position of the lithium cobalt oxide on the electrical chemical chip (b). 